\section{ADDITIONAL INFORMATION}

Publisher's note Springer Nature remains neutral with regard to jurisdictional claims in published maps and institutional affiliations.

\section{REFERENCES}

1. Dhawan BN, Cesselin F, Raghubir R, Reisine T, Bradley PB. et al. International union of pharmacology. XII. Classification of opioid receptors. Pharm Rev. 1996;48:567-92.

2. Stein C, Lang LJ. Peripheral mechanisms of opioid analgesia. Curr Opin Pharmacol. 2009:9:3-8.
3. Schmid CL, Kennedy NM, Ross NC, Lovell KM, Yue Z. et al. Bias factor and therapeutic window correlate to predict safer opioid analgesics. Cell. 2017;171:1165-75.

4. Stein C, Clark JD, Oh U, Vasko MR, Wilcox GL, Overland AC, Vanderah TW, Spencer RH. Peripheral mechanisms of pain and analgesia. Brain Res Rev. 2009;60:90-113.

5. Scherer PC, Zaccor NW, Neumann NM, Vasavda C, Barrow R, et al. TRPV1 is a physiological regulator of $\mu$-opioid receptors. Proc Natl Acad Sci USA. 2017;114:13561-6.

6. Basso L, Aboushousha R, Fan CY, Iftinca M, Melo H, Flynn R, Agosti F, Hollenberg MD, Thompson R, Bourinet E, Trang T, Altier C. TRPV1 promotes opioid analgesia during inflammation. Sci Signal. 2019;12:eaav0711

\title{
Towards objective definition of psychopathology in post-traumatic stress disorder
}

\author{
Amit Etkin $\mathbb{D}^{1,2,3}$ \\ Neuropsychopharmacology (2020) 45:226-227; https://doi.org/10.1038/s41386-019-0504-7
}

Owing to their reliance on imprecise clinical phenotypic definitions, current psychiatric diagnoses capture a broad range of neurobiological alterations across patients. The difficulties that arise from these definitions are particularly striking for posttraumatic stress disorder (PTSD). For example, the revision of its diagnostic criteria from DSM-IV to DSM5 resulted in only a 50\% overlap in case definition [1]. One way to overcome challenges inherent to these clinical definitions is to anchor patient definitions in objectively quantifiable measures [2].

To identify biologically and clinically meaningful PTSD subtypes, we began with the perspective that cognitive task behavior may be a particularly useful way to anchor patient phenotypes so that they are both objective and face-valid [3]. Within cognition, verbal memory is the domain most impaired in PTSD patients on average [4]. We therefore treated verbal memory in a normative perspective, dividing patients based on whether they performed within or outside the healthy norm, and examined resting-state functional magnetic resonance imaging (fMRI) network connectivity to understand mechanisms involved with differences in memory [3]. This is akin to a typical medical test, which are often framed within a normative perspective. We found, and then replicated (total $N=357$ ), that after correction for multiple comparisons, connectivity in one brain system (the ventral attention network; VAN) was reduced only in PTSD patients with impaired verbal memory, relative to either controls or patients with intact memory.

Critically, moreover, memory and VAN connectivity predicted treatment outcome, thus demonstrating clinical relevance, despite the discovery of the memory-VAN connection coming out of a mechanistic characterization rather than one primarily targeting treatment prediction [3]. Patients in one of the samples went on to receive either prolonged exposure psychotherapy, a gold-standard treatment for PTSD, or a wait list intervention control. Those patients with impaired memory and VAN connectivity failed to respond to prolonged exposure (and did not differ in the wait list arm), whereas those without both abnormalities responded well. Finally, to understand how these insights may be useful in driving new therapeutics, we used simultaneous non-invasive transcranial magnetic stimulation and electroencephalography (TMS/EEG) to map the brain's response to single TMS pulses at various locations and implicated a region in the right prefrontal cortex [3].

These results suggest that by anchoring on an objective measure (i.e., verbal memory), clinically and mechanistically meaningful biological differences can be observed and replicated. The TMS findings further suggest an avenue for developing novel interventions for memory-VAN impaired patients, by targeting the right prefrontal cortex.

More broadly, these findings open up a path for transcending traditional clinical phenomenology and grounding clinically meaningful case definition in observable biomarkers. To ultimately impact clinical care, we anticipate that tools such as EEG (a cheaper and more clinic-ready tool than fMRI) and machine learning (to make relevant brain signatures more robust) will be required. Nonetheless, our results suggest that it is more a question of how, rather than whether, these types of biomarkers could transform diagnosis and treatment in psychiatry.

\section{FUNDING AND DISCLOSURE}

Dr. Etkin was funded by NIH grant DP1 MH116506. Dr. Etkin holds equity in Mindstrong Health, Akili Interactive, and Sizung for unrelated work.

\section{ADDITIONAL INFORMATION}

Publisher's note Springer Nature remains neutral with regard to jurisdictional claims in published maps and institutional affiliations.

\section{REFERENCES}

1. Stein DJ, McLaughlin KA, Koenen KC, Atwoli L, Friedman MJ, Hill ED, et al. DSM-5 and ICD-11 definitions of posttraumatic stress disorder: investigating "narrow" and "broad" approaches. Depress Anxiety. 2014;31:494-505.

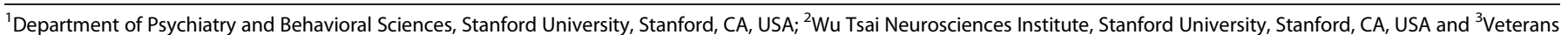
Affairs Palo Alto Healthcare System, Sierra Pacific Mental Illness, Research, Educatioand Clinical Center (MIRECC), Palo Alto, CA, USA

Correspondence: Amit Etkin (amitetkin@stanford.edu)

Published online: 5 September 2019 
2. Drysdale AT, Grosenick L, Downar J, Dunlop K, Mansouri F, Meng Y, et al. Restingstate connectivity biomarkers define neurophysiological subtypes of depression. Nat Med. 2017;23:28-38.

3. Etkin A, Maron-Katz A, Wu W, Fonzo GA, Huemer J, Vertes PE, et al. Using fMRI connectivity to define a treatment-resistant form of post-traumatic stress disorder. Sci Transl Med. 2019;11:eaal3236.
4. Scott JC, Matt GE, Wrocklage KM, Crnich C, Jordan J, Southwick SM, et al. A quantitative meta-analysis of neurocognitive functioning in posttraumatic stress disorder. Psychol Bull. 2015;141:105-40.

\title{
Linking actions with their consequences within the ventrolateral orbital cortex
}

\author{
Dan C. $\mathrm{Li}^{1}$ and Shannon L. Gourley (iD) ${ }^{1,2}$ \\ Neuropsychopharmacology (2020) 45:227-228; https://doi.org/10.1038/s41386-019-0498-1
}

Humans and rodents can associate actions with their outcomes and modify expectations when associations change. These cognitive adaptions accommodate change and presumably optimize decision-making. For example, we might modify our driving route when construction blocks our path or abstain from alcohol when we need to drive, expecting that both actions will deliver us safely home. Across species, medial prefrontal cortical regions are involved in linking actions with valued outcomes, but contributions of the orbitofrontal cortex remain contentious. One issue is that the orbitofrontal cortex occupies a large territory, yet is sometimes treated as a homologous structure. A related concern is the assumption that ventromedial subregions, like lateral regions, specialize in stimulus-outcome associations (linking cues, rather than actions, with likely outcomes) agnostic to action-outcome associations. Nevertheless, poor or aberrant decision-making is commonplace in neuropsychiatric illnesses, necessitating a full dissection of how action-outcome associations form, update, and solidify.

Parkes et al. [1] made important in-roads in resolving controversies. Rats were trained to associate two actions with a single food reward; then the actions were paired with different, unique rewards in the days preceding a devaluation test. Inactivation of the ventrolateral orbitofrontal cortex (VLO) either during the final training days or during the subsequent probe test blocked the ability of rats to choose actions based on the value of respective rewards. Meanwhile, VLO inactivation had no effect when action-outcome contingencies had not changed during training. In another investigation, inactivation of the VLO immediately following the violation of a familiar action-outcome association in mice occluded optimal responding in a later test, even when the VLO was back "on-line" [2]. Thus, the VLO appears necessary for stabilizing newly formed or updated action-outcome associations, which then guide future behavior.

Notably, linking actions with their outcomes involves dendritic spine plasticity on excitatory neurons in the VLO [2]. Specifically, updating action-outcome expectations reduces thin-type dendritic spines, considered immature, on layer $\mathrm{V}$ neurons. Meanwhile, the proportion of mushroom-shaped spines, considered mature, increases, potentially solidifying newly modified action -outcome associations to optimize future decision-making. Remarkably, inactivating VLO neurons upon the violation of familiar action-outcome associations not only blocks response updating, but also inhibits dendritic spine plasticity in an activitydependent manner [2]. These patterns strongly suggest that dendritic spine plasticity on excitatory VLO neurons is necessary for forming action-outcome associations, consistent with evidence that orbitofrontal neurons are capable of forming and maintaining long-term reward-related memory to support behavioral adaptations [3].

Orbitofrontal neurons display a rich diversity of functionally distinct populations based on input/output patterns, many of which make unique contributions to flexible decision-making $[3,4]$. A key question is thus: What inputs to the VLO help to form/update action-outcome associations? Are these inputs distinct from those supporting other associations, e.g., stimulus - outcome associations? Basolateral amygdala (BLA) projections are one candidate. BLA lesions alter the reward-related coding properties of orbitofrontal neurons [5], and BLA $\rightarrow$ orbitofrontal cortical connections appear necessary for certain forms of reinforcement learning [4], including in primates ([6] and references therein). Whether and how these "bottom-up" connections are involved in forming action-outcome associations should be resolved.

\section{FUNDING AND DISCLOSURE}

DCL and SLG are supported by the National Institute of Mental Health and National Institute on Drug Abuse at the National Institutes of Health (grant numbers 044297, 117103, 117873). The authors declare no competing interests.

\section{ADDITIONAL INFORMATION}

Publisher's note: Springer Nature remains neutral with regard to jurisdictional claims in published maps and institutional affiliations.

\footnotetext{
${ }^{1}$ Graduate Training Program in Neuroscience, Departments of Pediatrics and Psychiatry, Emory University School of Medicine, Yerkes National Primate Research Center, Emory University, 954 Gatewood Road NE, Atlanta, GA 30329, USA and ${ }^{2}$ Graduate Training Program in Molecular and Systems Pharmacology, Emory University, Atlanta, GA 30329, USA Correspondence: Shannon L. Gourley (shannon.l.gourley@emory.edu)
}

Received: 17 July 2019 Revised: 19 August 2019 Accepted: 20 August 2019

Published online: 2 September 2019 